\title{
BMJ Open Multidimensional instruments with an integral approach to identify frailty in community-dwelling people: protocol for a systematic psychometric review
}

\author{
Juan José Zamora-Sánchez (10 , ${ }^{1}$ Vicente Gea-Caballero, ${ }^{2}$ Iván Julián-Rochina, ${ }^{3}$ \\ Lina Jódar-Fernández, ${ }^{1}$ Iris Lumillo-Gutiérrez, ${ }^{1}$ Meritxell Sastre-Rus, ${ }^{4}$ \\ Ana-María Urpí-Fernández, ${ }^{1}$ Edurne Zabaleta-del-Olmo (i) ${ }^{5}$
}

To cite: Zamora-

Sánchez JJ, Gea-Caballero V, Julián-Rochina I, et al. Multidimensional instruments with an integral approach to identify frailty in communitydwelling people: protocol for a systematic psychometric review. BMJ Open 2019;9:e033160. doi:10.1136/ bmjopen-2019-033160

- Prepublication history for this paper is available online. To view these files, please visit the journal online (http://dx.doi org/10.1136/bmjopen-2019033160).

Received 23 July 2019 Revised 18 November 2019 Accepted 18 November 2019

Check for updates

(C) Author(s) (or their employer(s)) 2019. Re-use permitted under CC BY-NC. No commercial re-use. See rights and permissions. Published by BMJ.

${ }^{1}$ Institut Català de la Salut, Barcelona, Spain

${ }^{2}$ Escuela Universitaria de Enfermería La Fe, Valencia, Spain

${ }^{3}$ Universitat de València, Valencia, Spain

${ }^{4}$ Escola Universitària d'Infermeria Gimbernat i Tomàs Cerdà, Barcelona, Spain

${ }^{5}$ Institut de Recerca en Atenció Primària Jordi Gol, Barcelona, Spain

Correspondence to Juan José Zamora-Sánchez; jjzamora.bcn.ics@gencat.cat

\section{ABSTRACT}

Introduction An increasing number of investigations highlight the complex nature of frailty; therefore, the use of multidimensional assessment instruments could be useful in clinical decision-making. Frail people are found mainly in the community setting which is why this is the ideal environment for early screening and intervention. For this purpose, it is necessary to have valid, time-effective and easy-to-use frailty assessment instruments. The aim of this review is to critically appraise, compare and summarise the quality of the measurement properties of all multidimensional instruments with an integral approach to identify frailty in community-dwelling people.

Methods and analysis Medline, Psychological Information Database (PsycINF0) and Cumulative Index to Nursing and Allied Health Literature (CINAHL) will be searched from their inception dates. We will also conduct searches in databases of grey literature. No limits will be applied for language. A highly sensitive validated search filter will be used for finding studies on measurement properties. An additional search including the names of the instruments found in the initial search will also be undertaken. Studies aiming at the development of a measurement instrument, the evaluation of one or more measurement properties or the evaluation of its interpretability will be included. The instrument should have an integral approach (physical, psychological and social) and it should measure all three domains. The context of use should be a community setting. Two reviewers independently will screen the references and assess the risk of bias by consensus-based standards for the selection of health measurement instruments checklist. To assess the overall evidence for the measurement properties of the identified instruments, the results of the different studies, adjusted for their methodological quality, will be combined.

Ethics and dissemination Ethical approval and patient consent are not required as this is a psychometric review based on published studies. The results of this review will be disseminated at conferences and published in an international peer-reviewed journal. PROSPERO registration number CRD42019120212.

\section{Strengths and limitations of this study}

- The review will be carried out using the best practices in psychometric reviews (consensus-based standards for the selection of health measurement instruments guidelines).

- A comprehensive literature search strategy has been planned.

- We will not use language restrictions in the search strategy.

- In this review, measurement properties, interpretability and feasibility of the measurement instruments will be assessed.

- This work will be helpful to identify the most valid, time-effective and easy-to-use frailty assessment instruments in community-dwelling people.

\section{INTRODUCTION}

Global ageing is contributing to an increase in the number of people with multimorbidity and complex care needs. ${ }^{1}$ In this regard, the application of the concept of frailty can be useful in order to deal with these problems. People in fragile state have a loss of functional reserve and an increase in vulnerability to stressors. Frailty is associated with several important adverse health outcomes such as increase in disability and in mortality, falls, institutionalisation, hospitalisation, lower quality of life and a greater use of healthcare. ${ }^{2-5}$

Frailty is potentially preventable and strategies to prevent and stop its progression are essential. A great amount of research has been carried out in order to identify who can benefit from these strategies, most of it focusing on the development of frailty measurements to objectively quantify this concept. ${ }^{6-9}$ Nevertheless, the concept of frailty is in constant evolution and there is a wide debate and lack of agreement on its 
conceptual and operational definition, so there is broad variability with respect to the conceptual frameworks and theoretical perspectives of these instruments. ${ }^{6710}$

The two most commonly used frailty measurements are Fried's frailty phenotype ${ }^{11}$ and Rockwood and Mitnitski's Frailty Index. ${ }^{12}$ Fried's frailty phenotype is based on the physical characteristics of frailty by considering a person as frail if he/she presents three or more of the following criteria: unintentional weight loss, weakness, exhaustion, slowness and low physical activity. This frailty measurement has a unidimensional approach and does not include psychosocial components although there is broad consensus in the debate regarding the multidimensional nature of frailty, with physical and psychosocial factors involved in its development. ${ }^{71013-15}$ Likewise, if frailty is measured only in terms of physical losses, there is the danger of carrying out prevention and management strategies focused solely on these aspects. ${ }^{10}$ The Rockwood and Mitnitski's Frailty Index is based on biological causative theory and involves the accumulation of 30 or more comorbidities, symptoms, diseases, disabilities or any health deficiency. ${ }^{12}$ Its rationale points out that the greater the number of health deficits, the higher the frailty. ${ }^{16}$ This index incorporates the multidimensional nature of frailty, but its calculation can be time consuming so it is not popular among clinicians. ${ }^{6} 17$ Moreover, given the complex nature of frailty and in the absence of a consensual definition, the components of frailty vary considerably between the different measurement instruments. ${ }^{18}$ Frailty, disability for activities of daily living and multimorbidity are overlapped but there is some agreement that these concepts should be separated. ${ }^{14} 19$ Disability for activities of daily living should be seen as an adverse outcome of frailty and multimorbidity as an antecedent. ${ }^{14}$ For this reason, it is suggested that these components should be approached separately, since on one hand they are related, on the other their management is different and considering them as frailty components may confuse the interventions designed to prevent and mitigate frailty. ${ }^{671418}$

Frail people are found mainly in the community setting which is why this is the ideal environment for early screening and intervention. ${ }^{2021}$ These early activities can help prevent, decrease and delay adverse health consequences for people and their families or caregivers. Healthcare in this setting should be integral, considering the physical, psychosocial and environmental aspects. ${ }^{7}$ Therefore, multidimensional instruments with an integral and community-based approach may be more useful than those based on acute care for this preventive purpose. However, most of these instruments have been evaluated from the perspective of their predictive value of adverse outcomes, and evaluation of their suitability as tools for clinical decision-making and as an intervention target is scarce. ${ }^{22}$ Furthermore, measurement properties of an instrument may be affected by the measurement setting, so an instrument suitable for a context of use might not be valid for another. ${ }^{23}$ On the other hand, frailty identification in community-dwelling people can involve a resource-intensive process. That is why, clinicians, mostly family physicians and community nurses, express the need for time-effective and easy-to-use instruments as well as valid and reliable frailty assessment. ${ }^{24} 25$ That is why, it is necessary to identify which are the most suitable instruments for community care.

An umbrella review of frailty screening tools ${ }^{26}$ identifies three reviews which included community-dwelling people. ${ }^{27-29}$ There are also systematic reviews focused on primary care or public health settings. ${ }^{90-32}$ However, none of these reviews use a specific methodology for systematic psychometric reviews of measurement instruments. Likewise, some of these reviews are mainly focused on the use of the instruments and not on their quality. ${ }^{27} 31$ Besides, these reviews are not aimed at identifying frailty measurement instruments based on a multidimensional and integral conceptual model. In addition, comprehensive methodological guideline for systematic reviews of measurement instruments has recently been developed by the Consensus-based Standards for the selection of health Measurement INstruments (COSMIN). ${ }^{33-35}$ The use of this guideline allows selecting the best measurement instrument in a methodologically sound way.

For all these reasons, we plan to carry out a systematic psychometric review on multidimensional instruments to identify frailty in community-dwelling people according to COSMIN methodology. The aim of this review is to critically appraise, compare and summarise the quality of the measurement properties of all multidimensional instruments with an integral approach to identify frailty in community-dwelling people. We will seek to answer the following question: What are the most suitable multidimensional measurement instruments with an integral approach to identify frailty among community-dwelling people?

\section{METHODS AND ANALYSIS}

We will perform a systematic psychometric review according to the procedure proposed by COSMIN initiative. ${ }^{33-35}$ The review protocol adheres to the Preferred Reporting Items for Systematic Review and Meta-Analysis Protocols (PRISMA-P) statement. ${ }^{36}$

\section{Eligibility criteria}

We will apply the following eligibility criteria.

\section{Study designs}

The study should be aimed at developing a measurement instrument, evaluating one or more measurement properties or its interpretability and feasibility. We will exclude studies that only use the measurement instrument as an outcome measurement instrument (eg, randomised controlled trials) or studies in which the instrument is used in a validation study of another instrument. Likewise, as recommended by COSMIN guidelines, we will 
exclude congress abstracts because often very limited information on the design of the study is found in them.

\section{Construct of interest}

The instrument should have an integral approach according to the following definition of frailty: 'a dynamic state affecting an individual who experiences losses in one or more domains of human functioning (physical, psychological or social), which is caused by the influence of a range of variables and which increases the risk of adverse outcomes' ${ }^{14}$ We will select only instruments which include measurements of all three domains: physical domain (nutrition, mobility, physical activity, strength, endurance, balance, sensory functions), psychological domain (cognition, mood and coping) and social domain (social relations and social support). This eligibility criterion is based on the need to provide care focuses on the whole person since focusing on a single domain can lead to fragmentation of care.$^{10}$ Likewise, if frailty is measured in only one domain, there is a danger of carrying out prevention and management strategies focused on a single domain. Moreover, if only one domain is measured, there is a risk that frail people will not be identified because the domains not evaluated could be affected.

\section{Study sample}

The study sample should represent community-dwelling people (at least $50 \%$ of the sample) of any age (in order to identify the most age-appropriate instruments).

\section{Context of use}

The measurement setting should be a community setting, for instance, adult day care centres, home or primary healthcare centres.

\section{Information sources}

We will perform a comprehensive literature search in Medline (via PubMed), Cumulative Index to Nursing and Allied Health Literature (CINAHL) (via Elton Bryson Stephens Company (EBSCO)) and Psychological Information Database (PsycINFO). We will also conduct searches in the databases of grey literature (OpenGrey and Grey Literature Report). Databases will be searched from their inception and language restrictions will not be applied. We will not use language restrictions in the search strategy. Reviewers are proficient in English, French and Spanish; if any selected article is written in other languages, they will have financial resources to translate it. We will check the reference lists of included studies and used citation tracking resources in order to identify additional relevant studies. Finally, we will perform an additional search including the names of the instruments which are found in the initial search.

\section{Search strategy}

The search will contain search terms related to the following aspects: (1) construct of interest: frailty; (2) target population: community-dwelling people; (3) measurement properties: a highly sensitive validated

\section{Box 1 Draft Medline (via PubMed) search strategy}

1. "Frailty" $[(\mathrm{MH}]) \mathrm{OR}$ "Frail Elderly"[(MH])

2. Frail*

3. \#1 OR \#2

4. "Primary Health Care" $[(\mathrm{MH}])$ OR "Ambulatory Care" $[(\mathrm{MH}]) \quad \mathrm{OR}$ "Outpatients"[(MH]) OR "Home Care Services"[(MH]) OR "Nursing Homes" $[(\mathrm{MH}])$ OR "Homes for the Aged" $[(\mathrm{MH}])$ OR "Home Care Services"[(MH]).

5. "Primary care" OR "Primary Health care" OR "Primary Healthcare" OR "Ambulatory care" OR outpatient* OR "community-dwelling" OR "community dwelling" OR community OR home* OR resident* OR domestic OR domiciliary.

6. \#4 OR \#5

7. "Filter for measurement properties" by Terwee et al. ${ }^{37}$

8. \#3 AND \#6 AND \#7

search filter for finding relevant studies on measurement properties. ${ }^{37}$ A draft Medline (via PubMed) strategy is described in box 1 .

\section{Select abstracts and full-text articles}

Literature search results will be uploaded to Rayyan QCRI, a free web application that helps collaboration among reviewers during study selection process. ${ }^{38}$ Reviewers will independently screen the titles and abstracts yielded by the search against the eligibility criteria. Full-text article for all records that will meet the eligibility criteria or where there is any doubt will be obtained. Subsequently, two reviewers will screen the full-text articles and decide which of them meet the eligibility criteria. If necessary, we will contact the authors to resolve doubts about eligibility. Differences will be resolved by discussion and if consensus between the two reviewers cannot be reached, a third reviewer will be consulted. We will record the reasons for exclusion.

\section{Evaluate the measurement properties}

The methodological quality of every single study on a measurement property will be assessed using the COSMIN risk of bias checklist. ${ }^{33}$ This checklist has 10 boxes to assess the following measurement properties: (1) instrument development; (2) content validity; (3) structural validity; (4) internal consistency; (5) crosscultural validity/measurement invariance: (6) reliability; (7) measurement error; (8) criterion validity; (9) hypotheses testing for construct validity and (10) responsiveness. Reviewers will determine which measurement properties are assessed in each article and each measurement property will be assessed separately, using the corresponding COSMIN box. Each measurement property will be rated as very good, adequate, doubtful or inadequate quality. Subsequently, the result of each study on each measurement property will be rated against the criteria for good measurement properties, ${ }^{39} 40$ and each result will be rated as either sufficient $(+)$, insufficient $(-)$ or indeterminate (?). We will use the Excel file provided on the COSMIN website for data entry and we will calculate overall ratings 
(https://cosmin.nl/wp-content/uploads/Scoring-formCOSMIN-boxes_april_final.xlsx). These judgements will be made independently by two review authors. Any disagreement will be resolved by discussion and if it is not resolved, a third reviewer will be consulted. Data extraction will be done by two reviewers independently and we will use the overview tables available on COSMIN website (https://cosmin.nl/wp-content/uploads/Emptytables.docx).

\section{Describe interpretability and feasibility}

We will also extract data on the characteristics of the instrument (characteristics of the included samples and results on the measurement properties) and on information about interpretability and feasibility of its scores (distribution of scores, floor and ceiling effects, purpose and context of use, completion time, cost, length, type and ease administration, and so on). This information will be used to decide if the different studies are sufficiently similar to be pooled or qualitatively summarised.

\section{Data synthesis and confidence in cumulative evidence}

If the results of all available studies per measurement property are consistent, the results of studies will be quantitatively pooled or qualitatively summarised, and compared against the criteria for good measurement properties. The quality of evidence will be graded using a modified Grading of Recommendations Assessment, Development, and Evaluation approach as a high, moderate, low and very low evidence. ${ }^{34}$ If the results are inconsistent, we will use several strategies: (1) find explanations and summarise per subgroup, (2) do not summarise the results and do not grade the evidence, or (3) base the conclusion on the majority on consistent results, and downgrade for inconsistency.

\section{Formulate recommendations}

Finally, we will formulate recommendations on the most suitable instruments ${ }^{340}$ into three categories:

A. measurement instrument with evidence for sufficient content validity and at least low-quality evidence for sufficient internal consistency. This instrument can be recommended for use and results obtained with this instrument can be trusted.

B. Measurement instrument categorised not in A or C. This instrument has potential to be recommended for use, but it requires further research to assess its quality.

C. Measurement instrument with high-quality evidence for an insufficient measurement property. This instrument should not be recommended for use.

These recommendations will be based on the evaluation of the measurement properties and on interpretability and feasibility aspects.

\section{Patient and public involvement}

Patients were not invited to contribute to the writing or editing of this protocol for readability or accuracy. At this time, this systematic psychometric review will be done without patient involvement.

\section{ETHICS AND DISSEMINATION}

Ethical approval and patient consent are not required as this is a review based on published studies. The review will be carried out using the best practices in systematic psychometric reviews. ${ }^{33-35}$ Results will be reported according to COSMIN and PRISMA) guidelines ${ }^{41}$ and will be disseminated at conferences and published in an international peer-reviewed journal.

Twitter Edurne Zabaleta-del-Olmo @EdurneZabaleta

Acknowledgements The authors appreciate the review of the English text by Patryk Bialoskorski, MA.

Contributors J-JZ-S and EZ-d-0 contributed to the conception and design of the review, drafted the protocol and designed the search strategy. VG-C, IJ-R, LJ-F, IL-G, MS-R and A-MU-F provided critical insights and revised the protocol. J-JZ-S registered the protocol review in the PROSPERO database. All authors have read and approved the final submitted version of this protocol. J-JZ-S is the guarantor of this systematic psychometric review.

Funding This systematic psychometric review was supported by the Health Department grant number (SLT008/18/00011) from the Generalitat of Catalunya (Spain) and by a grant from the Territorial Management of Barcelona of the Institut Català de la Salut (Catalan Institute of Health) to the first author in the 2018 edition.

Disclaimer The funders had no role in review design, decision to publish or preparation of this protocol.

Competing interests None declared.

Patient consent for publication Not required.

Provenance and peer review Not commissioned; externally peer reviewed.

Open access This is an open access article distributed in accordance with the Creative Commons Attribution Non Commercial (CC BY-NC 4.0) license, which permits others to distribute, remix, adapt, build upon this work non-commercially, and license their derivative works on different terms, provided the original work is properly cited, appropriate credit is given, any changes made indicated, and the use is non-commercial. See: http://creativecommons.org/licenses/by-nc/4.0/.

\section{ORCID iDs}

Juan José Zamora-Sánchez http://orcid.org/0000-0002-3487-7789

Edurne Zabaleta-del-0lmo http://orcid.org/0000-0002-5072-8548

\section{REFERENCES}

1 Boehmer KR, Abu Dabrh AM, Gionfriddo MR, et al. Does the chronic care model meet the emerging needs of people living with multimorbidity? A systematic review and thematic synthesis. PLoS One 2018;13:e0190852.

2 Kojima G. Frailty as a predictor of nursing home placement among community-dwelling older adults. J Geriatr Phys Ther 2018;41:42-8.

3 Kojima G. Frailty as a predictor of disabilities among communitydwelling older people: a systematic review and meta-analysis. Disabil Rehabil 2017;39:1897-908.

4 Kojima G. Frailty as a predictor of hospitalisation among communitydwelling older people: a systematic review and meta-analysis. $J$ Epidemiol Community Health 2016;70:722-9.

5 Kojima G, lliffe S, Walters K. Frailty index as a predictor of mortality: a systematic review and meta-analysis. Age Ageing 2018;47:193-200.

6 Dent E, Kowal P, Hoogendijk EO. Frailty measurement in research and clinical practice: a review. Eur J Intern Med 2016;31:3-10.

7 Hoogendijk EO, Afilalo J, Ensrud KE, et al. Frailty: implications for clinical practice and public health. Lancet 2019;394:1365-75.

8 Bouillon K, Kivimaki M, Hamer M, et al. Measures of frailty in population-based studies: an overview. BMC Geriatr 2013;13:64.

9 Gilardi F, Capanna A, Ferraro M, et al. Frailty screening and assessment tools: a review of characteristics and use in public health. Ann Ig 2018;30:128-39.

10 Markle-Reid M, Browne G. Conceptualizations of frailty in relation to older adults. J Adv Nurs 2003;44:58-68.

11 Fried LP, Tangen CM, Walston J, et al. Frailty in older adults: evidence for a phenotype. J Gerontol Ser A Biol Sci Med Sci 2001;56:M146-57. 
12 Mitnitski AB, Mogilner AJ, Rockwood K. Accumulation of deficits as a proxy measure of aging. Sci World $J$ 2001;1:323-36.

13 Puts MTE, Toubasi S, Andrew MK, et al. Interventions to prevent or reduce the level of frailty in community-dwelling older adults: a scoping review of the literature and international policies. Age Ageing 2017;46:383-92.

14 Gobbens RJJ, Luijkx KG, Wijnen-Sponselee MT, et al. Towards an integral conceptual model of frailty. $J$ Nutr Health Aging 2010;14:175-81.

15 World Health Organization (WHO). World report on ageing and health 2015.

16 Searle SD, Mitnitski A, Gahbauer EA, et al. A standard procedure for creating a frailty index. BMC Geriatr 2008;8:24.

17 Hubbard RE, O'Mahony MS, Woodhouse KW. Characterising frailty in the clinical setting--a comparison of different approaches. Age Ageing 2008;38:115-9.

18 Xie B, Larson JL, Gonzalez R, et al. Components and indicators of frailty measures: a literature review. J Frailty Aging 2017;6:76-82.

19 Fried LP, Ferrucci L, Darer J, et al. Untangling the concepts of disability, frailty, and comorbidity: implications for improved targeting and care. J Gerontol Ser A Biol Sci Med Sci 2004;59:M255-63.

20 Collard RM, Boter H, Schoevers RA, et al. Prevalence of frailty in community-dwelling older persons: a systematic review. J Am Geriatr Soc 2012;60:1487-92.

21 Abbasi M, Rolfson D, Khera AS, et al. Identification and management of frailty in the primary care setting. Can Med Assoc J 2018;190:E1134-40.

22 Buta BJ, Walston JD, Godino JG, et al. Frailty assessment instruments: systematic characterization of the uses and contexts of highly-cited instruments. Ageing Res Rev 2016;26:53-61.

23 Walton MK, Powers JH, Hobart J, et al. Clinical outcome assessments: conceptual Foundation-Report of the ISPOR clinical outcomes assessment - emerging good practices for outcomes research Task force. Value Heal 2015;18:741-52.

24 Britton $\mathrm{H}$. What are community nurses experiences of assessing frailty and assisting in planning subsequent interventions? $\mathrm{Br} J$ Community Nurs 2017;22:440-5.

25 De Lepeleire J, Degryse J, Illiffe S, et al. Family physicians need easy instruments for frailty. Age Ageing 2008;37:384.

26 Apóstolo J, Cooke R, Bobrowicz-Campos E, et al. Predicting risk and outcomes for frail older adults: an umbrella review of frailty screening tools. JBI Database Syst Rev Implement Reports 2017;15:1154-208.

27 Clegg A, Rogers L, Young J. Diagnostic test accuracy of simple instruments for identifying frailty in community-dwelling older people: a systematic review. Age Ageing 2015;44:148-52.
28 Drubbel I, Numans ME, Kranenburg G, et al. Screening for frailty in primary care: a systematic review of the psychometric properties of the frailty index in community-dwelling older people. BMC Geriatr 2014;14:27

29 Vermeulen J, Neyens JCL, van Rossum E, et al. Predicting ADL disability in community-dwelling elderly people using physical frailty indicators: a systematic review. BMC Geriatr 2011;11:33.

30 Sieliwonczyk E, Perkisas S, Vandewoude M. Frailty indexes, screening instruments and their application in Belgian primary care. Acta Clin Belg 2014;69:233-9.

31 Lee L, Patel T, Hillier LM, et al. Identifying frailty in primary care: a systematic review. Geriatr Gerontol Int 2017;27.

32 Pialoux T, Goyard J, Lesourd B. Screening tools for frailty in primary health care: a systematic review. Geriatr Gerontol Int 2012;12:189-97.

33 Mokkink LB, de Vet HCW, Prinsen CAC, et al. COSMIN risk of bias checklist for systematic reviews of patient-reported outcome measures. Qual Life Res 2018;27:1171-9.

34 Prinsen CAC, Mokkink LB, Bouter LM, et al. COSMIN guideline for systematic reviews of patient-reported outcome measures. Qual Life Res 2018;27:1147-57.

35 Terwee CB, Prinsen CAC, Chiarotto A, et al. COSMIN methodology for evaluating the content validity of patient-reported outcome measures: a Delphi study. Qual Life Res 2018;27:1159-70.

36 Moher D, Shamseer L, Clarke M, et al. Preferred reporting items for systematic review and meta-analysis protocols (PRISMA-P) 2015 statement. Syst Rev 2015;4:1.

37 Terwee CB, Jansma EP, Riphagen II, et al. Development of a methodological PubMed search filter for finding studies on measurement properties of measurement instruments. Qual Life Res 2009;18:1115-23.

38 Ouzzani M, Hammady H, Fedorowicz Z, et al. Rayyan-a web and mobile app for systematic reviews. Syst Rev 2016;5:210.

39 Terwee CB, Bot SDM, de Boer MR, et al. Quality criteria were proposed for measurement properties of health status questionnaires. J Clin Epidemiol 2007;60:34-42.

40 Prinsen CAC, Vohra S, Rose MR, et al. How to select outcome measurement instruments for outcomes included in a "Core Outcome Set" - a practical guideline. Trials 2016;17:449.

41 Moher D, Liberati A, Tetzlaff J, et al. Preferred reporting items for systematic reviews and meta-analyses: the PRISMA statement. $J$ Clin Epidemiol 2009;62:1006-12. 